\title{
On the Numerical Implementation of the Multiplicative Regularization in Microwave Imaging
}

\author{
Puyan Mojabi \\ University of Manitoba, Winnipeg, Manitoba, Canada
}

\begin{abstract}
We consider the widely-used weighted $L_{2}$ norm total variation multiplicative regularizer (MR) for both the Gauss-Newton inversion (GNI) and contrast source inversion (CSI) algorithms in microwave imaging (MWI). It is shown that the proper numerical implementation of the discretized MR operator is important for the GNI algorithm whereas the CSI algorithm is more robust with respect to different implementations of this MR. For the GNI algorithm, the MR operator should be discretized such that high spatial frequency components are not present in its nullspace, and also the resulting discrete operator is positive definite.
\end{abstract}

\section{Introduction}

In microwave imaging (MWI), one of the main challenges is how to treat the ill-posedness of the associated inverse scattering problem. To this end, regularization techniques are used, which consist of two main components: (i) the utilized regularization operator, and (ii) the method with which the weight of the regularization operator is determined. Several regularization techniques have been used in MWI, e.g., see [1]. One of the most successful MWI regularization methods, which is the focus of this paper, is the weighted $L_{2}$ norm total variation multiplicative regularization (MR) scheme, e.g., see [2, 3]. ${ }^{1}$ This particular MR has been successfully used with both the contrast source inversion (CSI) and the Gauss-Newton inversion (GNI) algorithms, resulting in the MR-CSI [2, 3] and MR-GNI [7, 8, 9] algorithms. The success of this MR lies in providing adaptive $^{2}$ and edge-preserving regularization $[1,3]$. Herein, we discuss one subtle, yet important, point regarding the numerical implementation of this MR. It will be discussed that the GNI performance is more sensitive to the numerical implementation of the MR operator as compared to CSI. As will be seen, this is related to the nullspace of the gradient operator as well as the positive-definiteness of the discretized MR operator. Herein, this numerical implementation is only discussed using pulse basis discretization for the $2 \mathrm{D}$ transverse magnetic microwave imaging.

\footnotetext{
${ }^{1}$ We note that other forms of MR, e.g., in the form of Tikhonov or shape/location regularization have also been used $[4,5,6]$. However, this paper is merely concerned with the weighted $L_{2}$ norm total variation MR.

${ }^{2}$ Adaptive regularization refers to the gradual decrease of the regularization weight in nonlinear inversion algorithms such as CSI and GNI.
}

\section{GNI versus CSI - A Review}

MWI is governed by two main equations: data and domain (state) equations [3]. The data equation maps the information within the imaging domain to the external measurement domain. On the other hand, the domain equation governs the interaction merely within the imaging domain. The GNI algorithm substitutes the domain equation into the data equation. The main advantage of the GNI approach is that it results in a cost functional that needs to be minimized merely over the unknown relative complex permittivity contrast profile $\chi$. However, the resulting GNI cost functional is ill-posed, and must be regularized. The CSI algorithm does not substitute the domain equation into the data equation, and instead uses the domain equation in the form of an additive regularization term. This provides a fundamental benefit for the CSI algorithm as it removes the necessity of having an extra layer of regularization for the CSI algorithm in many cases. However, it has been found that CSI can still benefit from an extra layer of regularization, in particular from the MR in some cases. On the other hand, the CSI cost functional needs to be minimized not only over $\chi$, but also over the contrast sources; thus, the number of unknowns increases as compared to GNI.

\section{Nullspace}

It is important that the nullspace ${ }^{3}$ of the regularization operator intersects trivially with that of the ill-posed operator. Assume that in a given iteration of the GNI algorithm, we deal with the following ill-posed equation $\mathbf{A} x=b$. Regularizing this equation with the regularization operator $\mathbf{R}$ results in the following $L_{2}$-norm minimization over the unknown $x$

$$
x_{\alpha}=\arg \min _{x}\left\{\|\mathbf{A} x-b\|^{2}+\alpha^{2}\left\|\mathbf{R}\left(x-x_{0}\right)\right\|^{2}\right\}
$$

where $x_{0}$ is an appropriate vector which can be, for example, a zero vector. In addition, $\alpha^{2} \in \mathbb{R}$ is an appropriate regularization weight. To ensure a unique regularized solution $x_{\alpha}$ for the above minimization, the intersection of the operators' nullspaces needs to be trivial: $\mathscr{N}(\mathbf{A}) \cap \mathscr{N}(\mathbf{R})=\emptyset[10]$. For example, for the case of standard Tikhonov regularization, we have $\mathbf{R}=\mathbf{I}$ where $\mathbf{I}$ is the identity operator. Noting that $\mathscr{N}(\mathbf{I})=\emptyset$, the above

\footnotetext{
${ }^{3}$ The nullspace of a given matrix $\mathbf{R}$, denoted by $\mathscr{N}(\mathbf{R})$, spans all the vectors that satisfy $\mathbf{R} x=0$.
} 


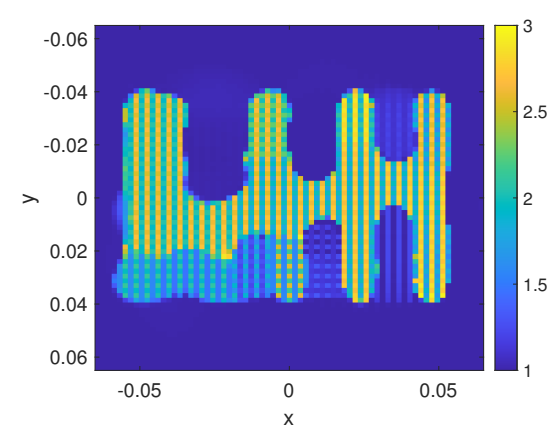

(a) MR-GNI - central

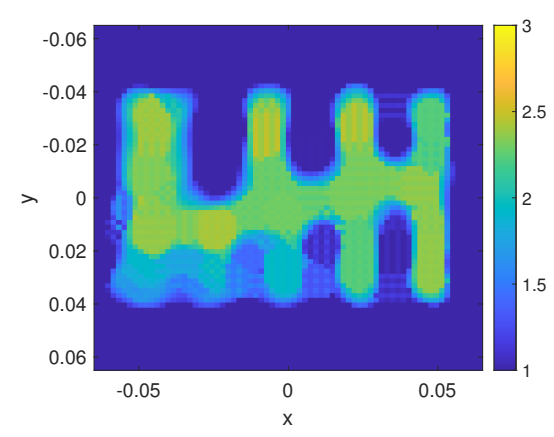

(c) MR-CSI - central

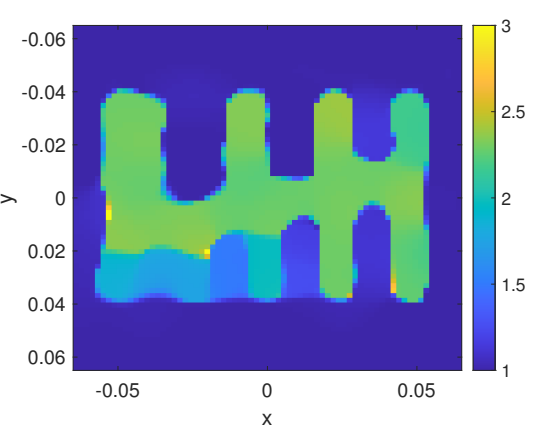

(b) MR-GNI - forward

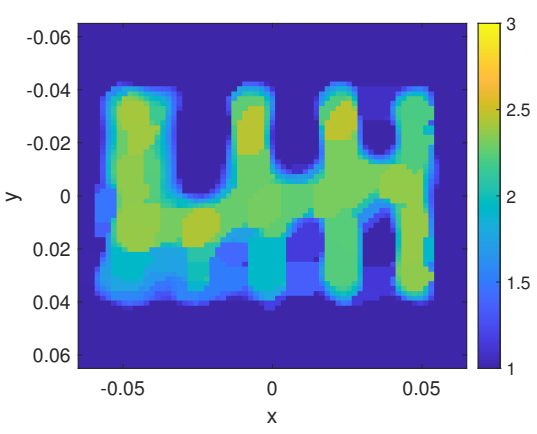

(d) MR-CSI - forward

Figure 1. Single-frequency $(5 \mathrm{GHz}$ ) inversion of the synthetic noiseless $E$-target dataset using (top row) MR-GNI and (bottom row) MR-CSI when the MR operator has been discretized using central (left) and forward (right) derivative approximations. Note the instability in the MR-GNI reconstruction under the central derivative approximation.

requirement is automatically satisfied. If the regularization operator has a non-trivial nullspace, we need to make sure that the intersection of its nullspace with the ill-posed operator $\mathbf{A}$ is trivial. To this end, the necessary condition is to ensure that $\mathscr{N}(\mathbf{R})$ does not have high spatial frequency components [11]. This is due to the fact that the ill-posed operator, due to its smoothing effects, often has a numerical nullspace containing high spatial frequency components. ${ }^{4}$ Therefore, if $\mathscr{N}(\mathbf{R})$ contains high spatial frequency components, the above requirement may not be satisfied.

Let us now consider the nullspace of the derivative (gradient) operator. In [11], it has been noted that for the 1D forward derivative approximation, the discretized derivative operator has a nullspace that is spanned by a constant vector. Therefore, this nullspace satisfies the necessary condition of not containing high spatial frequency components. On the other hand, the use of the 1D central derivative approximation results in a nullspace spanned by two vectors: a constant vector and a high spatial frequency vector. As noted in [11], the reason for having this high spatial fre-

\footnotetext{
${ }^{4}$ Not that $\mathbf{A} v_{i}=\sigma_{i} u_{i}$ where $u_{i}, v_{i}$, and $\sigma_{i}$ are the $i$ th left singular vector, the $i$ th right singular vector and the $i$ th singular value of $\mathbf{A}$, respectively. In ill-posed problems, for large $i$ indices, $\sigma_{i}$ is very small, and therefore $\mathbf{A} v_{i} \rightarrow 0$. Therefore, the numerical nullspace of $\mathbf{A}$ contains $v_{i}$ with large $i$ indices. Noting that $v_{i}$ with large $i$ indices correspond to high spatial frequency components [12], the numerical nullspace of $\mathbf{A}$ will have high spatial frequency components. Therefore, it is important that the nullspace of $\mathbf{R}$ does not have high spatial frequency components.
}

quency component is that the central derivative approximation only correlates every second point within the discretized domain. Therefore, due to having a high spatial frequency component, the central derivative approximation to the derivative operator is not appropriate to be used as a regularization operator.

Now, let us begin to see how this is related to the MR operator. The MR operator at the $n$th iteration of the inversion algorithm is $\mathscr{L}_{n}=-\nabla \cdot\left(b_{n}^{2} \nabla\right)$ where ' $\nabla$.' is the divergence operator and ' $\nabla$ ' is the gradient operator. The inhomogeneous real-valued weight $b_{n}^{2}$ is used to determine if the regularization operator should act locally as a smoothing operator (Laplacian) or as an edge-preserving operator. The important item to recognize is the presence of the gradient operator in the expression of $\mathscr{L}_{n}$. This paper does not thoroughly consider the nullspace of the whole operator $\mathscr{L}_{n}$. However, based on the nullspace of the derivative operator as explained above, we note that discretizing the MR operator using the central derivative approximation may cause instability in the inversion process. ${ }^{5}$ On the other hand, this is less likely to happen in the MR-CSI algorithm as the

\footnotetext{
${ }^{5}$ Based on our numerical experience, if the MR-GNI algorithm is terminated earlier, this instability issue with the central difference approximation may be mitigated. This is perhaps due to the fact that at later MR-GNI iterations the effect of the edge-preserving regularization, i.e., the gradient operator, is stronger, and therefore, the issue with its nullspace becomes more visible.
} 


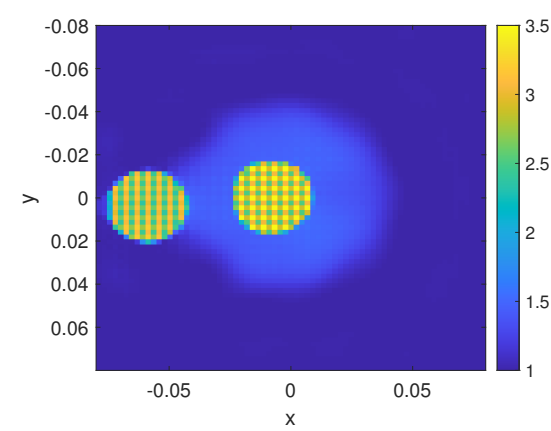

(a) MR-GNI - central

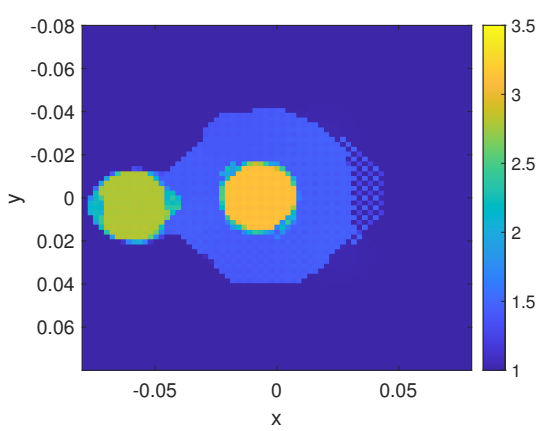

(c) MR-CSI - central

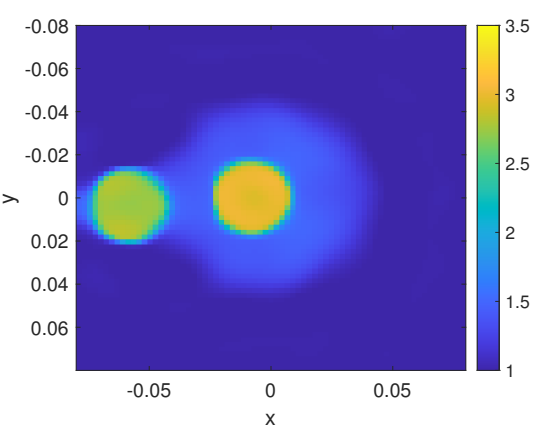

(b) MR-GNI - forward

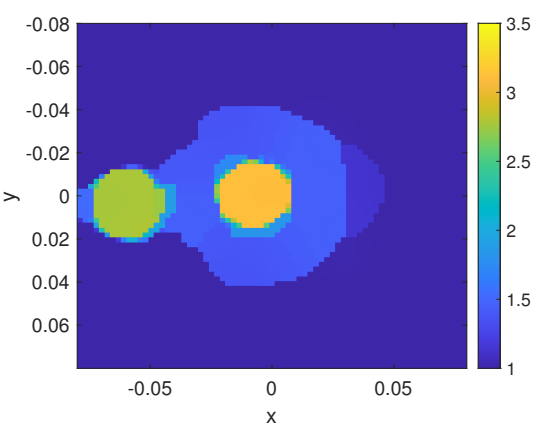

(d) MR-CSI - forward

Figure 2. Single-frequency (6 GHz) inversion of the experimental FoamTwinDielTM dataset using (top row) MR-GNI and (bottom row) MR-CSI when the MR operator has been discretized using central (left) and forward (right) derivative approximations. Note the instability in the MR-GNI reconstruction under the central derivative approximation.

MR-CSI algorithm has two layers of regularization (domain equation and the MR) whereas the MR-GNI algorithm has only one layer of regularization, i.e., the MR.

\section{Positive Definiteness}

The MR-GNI algorithm requires the calculation of the Hessian matrix $\mathbf{H}_{n}$ where $n$ denotes the iteration number. It is desired that the Hessian matrix be a positive definite matrix. Since one component of the Hessian matrix is the MR operator, $\mathscr{L}_{n}$, this requires the discretized implementation of the MR to be positive definite. One way to ensure that the discrete form of $\mathscr{L}_{n}$ is positive definite can be achieved as follows. When $\mathscr{L}_{n}$ operates on a vector of appropriate size, say $y$, the vector $y$ is first reshaped into a matrix with the same size as the 2D discretized imaging domain. Then, zeros will be added around this matrix as shown in Figure 3 . The derivative operators associated with $\mathscr{L}_{n}$ needs to consider this zero boundary condition when calculating the gradient for the marginal $y$ elements which are $y_{1 i}, y_{4 i}$, $y_{i 1}$, and $y_{i 4}$ for $\forall i$ for the case shown in Figure 3. This is based on what was presented in [13, Appendix E] in which it was shown that $\mathscr{L}_{n}$ is positive definite in the continuous domain under zero Dirichlet boundary condition. (This is a valid assumption in MWI since the complex permittivity contrast vanishes just outside the imaging domain.) Noting that the MR-CSI algorithm does not use the Hessian matrix, it does not require the discretized implementation of $\mathscr{L}_{n}$ to be positive definite.

\section{Results}

We consider two data sets: one synthetic and the other experimental data set. The synthetic data set was collected from the so-called $E$-target whose shape was originally considered in [14]. Similar to [15], the relative permittivity of the lossless E-target was set to 2.3, and the background medium was air. The synthetic data were collected from a $12 \times 12 \mathrm{~cm}^{2}$ domain discretized into $100 \times 100$ square pulses using 24 line sources at $5 \mathrm{GHz}$. The inversion was performed on a $13 \times 13 \mathrm{~cm}^{2}$ domain discretized into $71 \times 71$ square pulses. The MR-GNI reconstructions using the central and forward difference approximations of the MR have been shown in Figure 1(a) and (b) respectively. As can be seen, the central difference approximation of the MR is not quite stable. The MR-CSI reconstructions using the two implementations of the MR have also been shown in Figure 1(c) and (d). As can be seen, the nullspace issue associated with the central difference approximation is much less visible in the MR-CSI algorithm. Finally, we consider the FoamTwinDielTM experimental data set at $6 \mathrm{GHz}$ from Fresnel Institute [16], and invert it within a $16 \times 16 \mathrm{~cm}^{2}$ domain discretized into $71 \times 71$ square pulses. As shown in Figure 2, the MR-GNI algorithm using the central difference approximation is not perfectly stable; however, the rest of the algorithms and implementations are reasonably 


\begin{tabular}{|c|c|c|c|c|c|}
\hline 0 & 0 & 0 & 0 & 0 & 0 \\
\hline 0 & $y_{11}$ & $y_{12}$ & $y_{13}$ & $y_{14}$ & 0 \\
\hline 0 & $y_{21}$ & $y_{22}$ & $y_{23}$ & $y_{24}$ & 0 \\
\hline 0 & $y_{31}$ & $y_{32}$ & $y_{33}$ & $y_{34}$ & 0 \\
\hline 0 & $y_{41}$ & $y_{42}$ & $y_{43}$ & $y_{44}$ & 0 \\
\hline 0 & 0 & 0 & 0 & 0 & 0 \\
\hline
\end{tabular}

Figure 3. Considering an arbitrary vector, e.g., $y \in \mathbb{C}^{16}$, reshaping it into a matrix with the same size as the discretized imaging domain, and then adding zeros around this matrix to enforce the required zero Dirichlet boundary condition.

stable. Finally, we note that the MR-CSI algorithm also worked when $\mathscr{L}_{n}$ was not a positive definite operator.

\section{Conclusion}

The numerical implementation of the discretized MR operator in the GNI algorithm needs to be performed carefully. For the GNI algorithm, it needs to be checked that the resulting discrete MR operator avoids having high spatial frequency components in its nullspace and also is positive definite. On the other hand, the CSI algorithm seems to be more robust with respect to different numerical implementations of the MR partly due to the fact that the CSI cost functional is already regularized by the domain equation.

\section{Acknowledgements}

The financial support of NSERC Canada is acknowledged.

\section{References}

[1] P. Mojabi and J. LoVetri, "Overview and classification of some regularization techniques for the GaussNewton inversion method applied to inverse scattering problems," IEEE Transactions on Antennas and Propagation, vol. 57, no. 9, pp. 2658-2665, Sept 2009.

[2] A. Abubakar and P. M. van den Berg, "Iterative forward and inverse algorithms based on domain integral equations for three-dimensional electric and magnetic objects," J. Comput. Phys., vol. 195, pp. 236-262, 2004.

[3] A. Abubakar, P. M. van den Berg, and J. J. Mallorqui, "Imaging of biomedical data using a multiplicative regularized contrast source inversion method," IEEE Trans. Microwave Theory Tech., vol. 50, no. 7, pp. 1761-1777, July 2002.

[4] T. M. Habashy and A. Abubakar, "A general framework for constraint minimization for the inversion of electromagnetic measurements," Progress in Electromagnetics Research, vol. 46, pp. 265-312, 2004.
[5] N. Bayat and P. Mojabi, "Incorporating spatial priors in microwave imaging via multiplicative regularization," IEEE Transactions on Antennas and Propagation, vol. 68, no. 2, pp. 1107-1118, Feb 2020.

[6] P. Mojabi, J. LoVetri, and L. Shafai, "A multiplicative regularized Gauss-Newton inversion for shape and location reconstruction," IEEE Trans. Antennas Propag., vol. 59, no. 12, pp. 4790-4802, 2011.

[7] M. Ostadrahimi, P. Mojabi, A. Zakaria, J. LoVetri, and L. Shafai, "Enhancement of Gauss-Newton inversion method for biological tissue imaging," IEEE Transactions on Microwave Theory and Techniques, vol. 61, no. 9, pp. 3424-3434, Sept 2013.

[8] P. Mojabi and J. LoVetri, "Microwave biomedical imaging using the multiplicative regularized GaussNewton inversion," IEEE Antennas Wireless Propag. Lett., vol. 8, pp. 645-648, 2009.

[9] A. Abubakar, T. M. Habashy, G. Pan, and M. K. Li, "Application of the multiplicative regularized Gauss Newton algorithm for three-dimensional microwave imaging," IEEE Transactions on Antennas and Propagation, vol. 60, no. 5, pp. 2431-2441, May 2012.

[10] T. K. Jensen, "Stabilization algorithms for large-scale problems," Ph.D. dissertation, Technical University of Denmark, Kongens Lyngby, Denmark, 2006.

[11] J. Pedersen, Modular Algorithms for Large-Scale Total Variation Image Deblurring, Master's Thesis, Technical University of Denmark, Lyngby, 2005.

[12] P. C. Hansen, M. E. Kilmer, and R. H. Kjeldsen, "Exploiting residual information in the parameter choice for discrete ill-posed problems," BIT Numerical Mathematics, vol. 46, pp. 41-59, 2006.

[13] P. Mojabi, "Investigation and development of algorithms and techniques for microwave tomography," Ph.D. dissertation, University of Manitoba, Winnipeg, Manitoba, Canada, 2010.

[14] S. Semenov, R. Svenson, A. Bulyshev, A. Souvorov, A. Nazarov, Y. Sizov, V. Posukh, A. Pavlovsky, P. Repin, and G. Tatsis, "Spatial resolution of microwave tomography for detection of myocardial ischemia and infarction-experimental study on twodimensional models," IEEE Trans. Microwave Theory Tech., vol. 48, no. 4, pp. 538-544, Apr 2000.

[15] N. Bayat and P. Mojabi, "A mathematical framework to analyze the achievable resolution from microwave tomography," IEEE Trans. Antennas Propag., vol. 64, no. 6, pp. 1484-1489, April 2016.

[16] J.-M. Geffrin, P. Sabouroux, and C. Eyraud, "Free space experimental scattering database continuation: experimental set-up and measurement precision," Inverse Probl., vol. 21, pp. S117-S130, 2005. 